\title{
Properties of Rigid Polyurethane Foams with Blowing Agents and Catalysts
}

\author{
Kun Hyung ChoE, Dong Soo LeE, Won Jin SeO*, and Woo Nyon Kim*† \\ Department of Chemistry, Yonsei University, Shinchon-dong, Seoul 120-749, South Korea \\ *Department of Chemical \& Biological Engineering, Applied Rheology Center, Korea University, \\ Anam-dong, Seoul 136-701, South Korea
}

(Received August 11, 2003; Accepted March 9, 2004; Published May 15, 2004)

\begin{abstract}
Rigid polyurethane foams (PUFs) were prepared from polymeric 4,4-diphenylmethane diisocyanate (PMDI) and polyether polyol with amine catalyst. Water was used as the chemical blowing agent and, cyclopentane and hydrofluorocarbon (HFC) were used as the physical blowing agents. The kinetic rate of forming the PUF increased with the catalyst and water content. The cell size of the PUF sample decreased with increasing amount of the blowing and gelling catalysts. In the case of the PUF sample blown by water only, the amount of the blowing catalyst had no significant influence on the density and the compressive strength of the PUF sample. In the case of the PUF sample prepared with using HFC or cyclopentane as the blowing agent, however, as the amount of the blowing catalyst increased, the density and the compressive strength of the PUF sample increased. The PUF sample blown by the physical blowing agent had smaller cell size than the PUF sample blown by the chemical blowing agent, if compared at same density. The PUF sample blown by mixed blowing agent (cyclopentane/water $=7 / 3$, mole ratio) had lower thermal conductivity than the PUF samples blown by cyclopentane or water only, at the equal mole content of the blowing agents. This result suggests that the low thermal conductivity of the PUF sample blown by mixed blowing agent is due to the increase in the structural stability of the PUF foams. [DOI 10.1295/polymj.36.368]

KEY WORDS Kinetic Rate / Morphology / Chemical Blowing Agent / Physical Blowing Agent /
\end{abstract}

Polyurethane foams (PUFs) have been commercially used in wide variety of applications since the 1940s. These foams surround us in today's society, playing an important role in many industries and our daily lives. Especially rigid polyurethane foam is one of the most important thermal insulating materials used today for construction and for sole insulation in electric appliances like refrigerators, and freezers. ${ }^{1}$

Polyurethane foams generally consist of a solid polymer matrix and a gaseous phase formed by blowing agents. In some cases, more than one solid components are added in the solid phase as fillers or extenders. Foaming of polymeric materials is carried out by mechanical, chemical, or physical means. The most widely used method involves dispersing a gas throughout a fluid polymer phase and stabilizing the resultant foam. In most systems, foams are allowed to expand before stabilizing the system. ${ }^{2,3}$

The kinetic rate of forming PUF is increased by a catalyst or by raising temperature. ${ }^{2}$ The kinetic rate affects the cell morphology of PUF samples. In addition, the kinetic rate is the important factor in the manufacture of PUF. ${ }^{1,4}$ For design of a PUF, information of the kinetic rate and its effect on the properties of the PUF are necessary.

Thermal conductivity is the most important property for thermal insulating materials. The thermal con- ductivity of closed-cell foams depends mainly on the total gas content and the thermal conductivity of entrapped blowing gas inside the closed cells. In addition, the structure and structural stability of the foams also affect the thermal conductivity of foams. PUFs are formed by either physical blowing agents or chemical blowing agents. Physical blowing agent such as cyclopentane and HFC expand polyurethane (PU) by vaporization, and this reaction is endothermic. ${ }^{2,3} \mathrm{On}$ the contrary, chemical blowing agents such as water expand PU by carbon dioxide generated by the reaction between the blowing agent and isocyanate, and this reaction is exothermic., ${ }^{2,3}$

In this study, the effects of catalysts and blowing agents on the kinetic rate, the density, the compressive strength, the cell morphology, and thermal conductivity were investigated for rigid PUFs prepared from polymeric 4,4-diphenylmethane diisocyanate (PMDI) with a functionality of 2.9 and polyether polyol based on pentaerithritol. Distilled water was used as a chemical blowing agent, and cyclopentane and HFC were used as physical blowing agents. The effects of these catalysts on the density and the compressive strength were investigated. Furthermore, at the same blowing and polymerization conditions, the effects of the blowing agents on the cell size and the thermal conductivity of the PUF samples were examined. The cel-

${ }^{\dagger}$ To whom correspondence should be addressed (Tel: +82-2-3290-3296, Fax: +82-2-926-6102, E-mail: kimwn@korea.ac.kr). 
lular structure, the compressive strength, and the thermal conductivity of the PUF samples were studied with a field emission scanning electron microscopy (FE-SEM), a universal testing machine (UTM), and a heat flow meter, respectively.

\section{EXPERIMENTAL}

\section{Materials}

The materials used in this study were obtained from commercial sources. The polymeric MDI (PMDI) was supplied from BASF Korea Ltd. (Seoul, Korea). The polyether polyol based on pentaerithritol was supplied from KPC Co. (Ulsan, Korea). Distilled water was prepared in our laboratory. Cyclopentane and hydrofluorocarbon (HFC) with average molecular weight of $148 \mathrm{~g} / \mathrm{mol}$ were supplied from Finetec Co. (Ansung, Korea) and Solvay Korea Co. (Seoul, Korea), respectively. Triethylene diamine dissolved in dipropylene glycol to 33 weight $\%$ and pentamethyldiethylene triamine, which were used as gelling and blowing catalysts, respectively, were supplied from Air Products and Chemicals, Inc. (Allentown, PA, USA). Polysiloxane ether used as a surfactant was supplied from Osi Specialties, Inc. (USA). The characteristics of the materials are given in Table I. The polyether polyol was dried before use at $90^{\circ} \mathrm{C}$ for $24 \mathrm{~h}$ in a vacuum oven. The other chemicals were used as received.

\section{Sample Preparations}

Rigid PUF samples with various densities were synthesized with a "one-shot method" ${ }^{4-6}$ All chemicals were put into the reactor and mixed for $30 \mathrm{~s}$ at ambient condition with a brushless-type stirrer, which was ring guard propeller for protecting the wall and sensors in the reactor. The stirrer speed was set at
$3000 \mathrm{rpm}$ throughout the mixing. After mixing, the reactants were poured into an open mold $(250 \mathrm{~mm} \times$ $250 \mathrm{~mm} \times 100 \mathrm{~mm}$ ) to produce free-rise foams and were cured for 1 week at room temperature. Three replications for all the foams were executed.

To investigate the effects of the catalysts on the properties of the PUF samples, triethylenediamine (gelling catalyst) and pentamethyldiethylene triamine (blowing catalyst) were used. The amount of each catalyst was varied from 0.2 to 1.8 parts per 100 polyether polyol by weight (php) with the increment of $0.4 \mathrm{php}$ while the amounts of polyether polyol, polysiloxane ether, and distilled water as a chemical blowing agent were fixed at 100,1.0, and $1.0 \mathrm{php}$, respectively. The amount of PMDI required for the reaction with polyether polyol, and distilled water was calculated from their equivalent weights. For the completion of the reaction, excess PMDI ( $c a .5 \mathrm{wt} \%$; NCO/ $\mathrm{OH}=1.05$ ) was used.

To investigate the effects of the blowing agent on the properties of the PUF samples, distilled water, cyclopentane, and HFC were used. The amount of each catalyst was fixed at $0.6 \mathrm{php}$ and the amounts of polyether polyol and polysiloxane ether were fixed at 100 and $1.0 \mathrm{php}$, respectively. The amount of distilled water was varied from 1.0 to $3.5 \mathrm{php}$. For each PUF systems blown by various blowing agents, the amount of PMDI required for the reaction with polyether polyol and distilled water was calculated from their equivalent weights. For the completion of the reaction, excess PMDI ( $c a .5 \mathrm{wt} \%$; $\mathrm{NCO} / \mathrm{OH}=1.05$ ) was used.

\section{Measurements}

Density Measurement. Density was measured according to ASTM D1622. The size of the specimen was $30 \times 30 \times 30 \mathrm{~mm}$. Densities of five specimens were measured and averaged for each sample.

Table I. Characteristics of materials used in this study ${ }^{a}$

\begin{tabular}{|c|c|c|c|}
\hline Materials & Functionality & $\begin{array}{l}\text { Equivalent weight } \\
\qquad\left(\mathrm{g} \mathrm{mol}^{-1}\right)\end{array}$ & Comments \\
\hline $\begin{array}{l}\text { 4,4'-Diphenylmethane } \\
\text { diisocyanate }\end{array}$ & 2.9 & 133.5 & NCO content: $31.5 \%$ \\
\hline Polyether polyol & 4.0 & 117.0 & $\begin{array}{l}\text { OH value: } \\
480 \mathrm{mg} \mathrm{KOH} / \mathrm{g}\end{array}$ \\
\hline Distilled water & 2.0 & 9.0 & Chemical blowing agent \\
\hline Cyclopentane & - & - & $\begin{array}{l}\text { Physical blowing agent } \\
\text { (boiling point }=50.0^{\circ} \mathrm{C} \text { ) }\end{array}$ \\
\hline Hydrofluorocarbon & - & - & $\begin{array}{l}\text { Physical blowing agent } \\
\text { (boiling point }=40.2^{\circ} \mathrm{C} \text { ) }\end{array}$ \\
\hline Triethylene diamine & - & - & Gelling catalyst \\
\hline $\begin{array}{l}\text { Pentametyldiethylene } \\
\text { triamine }\end{array}$ & - & - & Blowing catalyst \\
\hline Polysiloxane ether & - & - & Surfactant \\
\hline
\end{tabular}

a Data from suppliers. 
Scanning Electron Microscopy. Morphology was observed with a HITACHI S-4300SE field emission scanning electron microscope. The samples were cryogenically fractured and gold coated before scanning. The accelerating voltage was $25 \mathrm{kV}$. The size of the cells of the PUF samples was measured with an Image-Pro Plus image analyzer and averaged.

Universal testing machine. Compressive strength was measured under ambient conditions with an Instron UTM (model 4467). A compressive test was performed according to ASTM D 1621. The size of the specimen was $30 \times 30 \times 30 \mathrm{~mm}$, and the speed of crosshead movement was $3.00 \mathrm{~mm} / \mathrm{min}$. The strengths of five specimens per sample were measured and averaged.

Thermal Conductivity Measurement. Thermal conductivity was measured with a Holometrix Micromet (model Lambda 2000) according to ASTM C 518. A sample was placed in the test section between the two plates which were maintained at different temperatures during the test. Upon achieving thermal equilibrium and establishing a uniform temperature gradient throughout the sample, the thermal conductivity of the PUF samples was determined. The size of the specimen was $300 \times 300 \times 50 \mathrm{~mm}$. The thermal conductivities of three specimens per sample were measured and averaged.

\section{RESULTS AND DISCUSSION}

\section{Effect of Catalysts}

The effect of catalysts on the kinetic rate of forming the PUF samples is shown in Figures 1 and 2. The kinetic rate of forming the PUF was assessed from the inverse of the characteristic times such as the cream time, gel time, and tack free time. The cream time is defined as the time when the color of mixed reactants becomes brightened. The gel time is the time when the stable spatial shape is formed. The tack free time is the time when the perfectly crosslinked PUF can be detached from the mold. ${ }^{6}$ From these figures, the kinetic rate of forming the PUF increases with the increase of the content of the gelling catalyst. In addition, the blowing catalyst promotes the blowing reaction, relatively, which was confirmed by the faster cream time, and the gelling catalyst promotes the gelling reaction, relatively, which was confirmed by the faster gel time and tack free time.

Figure 3 shows the cell morphologies of the PUF samples prepared with different amounts of the gelling catalysts: (a) $0.6 \mathrm{php}$ and (b) $1.8 \mathrm{php}$. The figure shows that the cell size of the PUF samples decreases from 307 to $132 \mu \mathrm{m}$ with the increase of the gelling catalyst. Although not shown in Figure 3, the blowing catalyst shows the similar behavior. Thus the kinetic rate

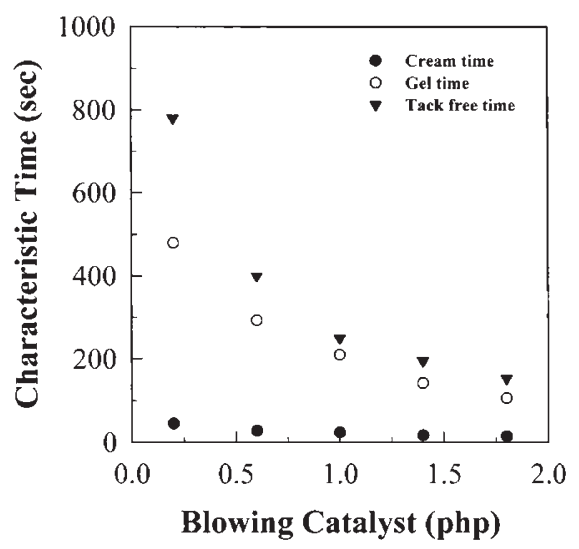

Figure 1. The dependence of the characteristic times for formation of PUF on the blowing catalyst content.

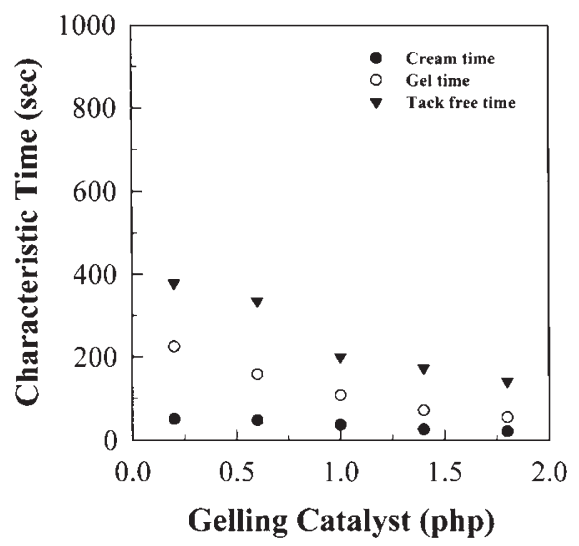

Figure 2. The dependence of the characteristic times for formation of PUF on the gelling catalyst content.

of forming gas bubbles increases with the catalyst content regardless of the types of the catalysts.

\section{Effect of Blowing Agents}

The effect of water content on the kinetic rate of the water blown PUF samples containing equal amount of the blowing and gelling catalyst is shown in Figures 4 and 5, respectively. From these figures, it is observed that the kinetic rate of forming PUF increases with the increase in water content regardless of the catalyst type. The increase in the kinetic rate with the water content is probably due to the increase in the temperature of the reactants by the heat of reaction evolved by the reaction between water and PMDI.

The effect of the blowing catalyst content on the density and the compressive strength is shown in Figures 6 and 7 for the PUF samples prepared with $\mathrm{HFC} /$ water (4/6 mole ratio) and with water only as the blowing agents, respectively. In Figure 6, it is seen that the density and the compressive strength of the PUF samples using HFC/water (4/6) as the blowing agents increase with the blowing catalyst content.

Generally polyurethane foams are produced by 


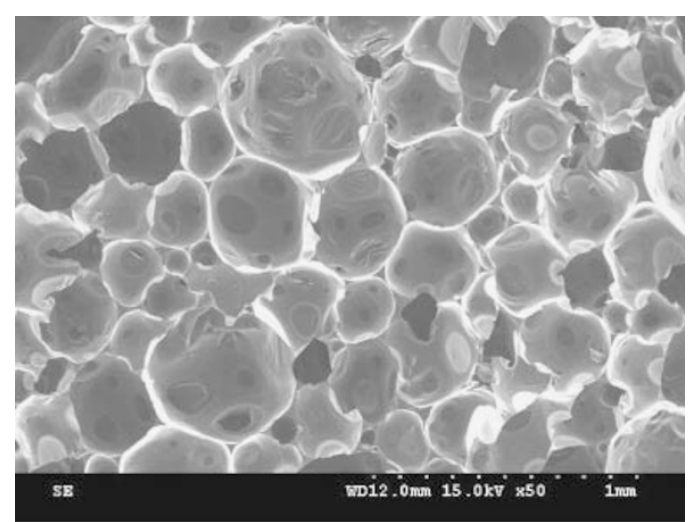

(a)

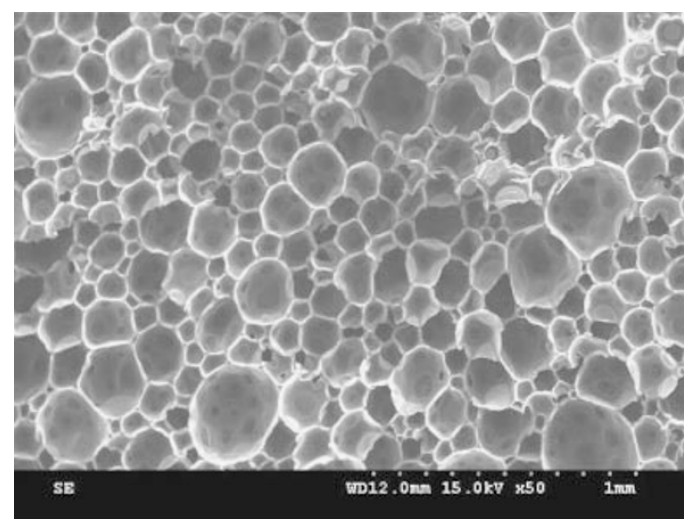

(b)

Figure 3. Scanning electron micrographs of the PUF samples with the gelling catalyst content: (a) $0.6 \mathrm{php}$, (b) $1.8 \mathrm{php}$.

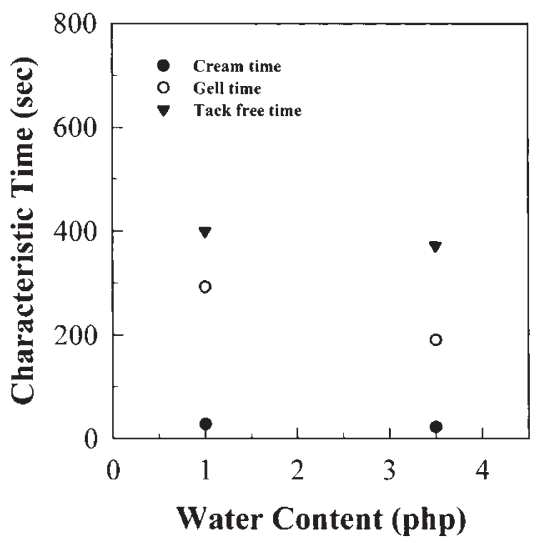

Figure 4. The dependence of the characteristic times of forming the PUF containing equal amount of the blowing catalyst on the water content.

forming a PU with concurrent a gas evolution process. Provided these two processes are balanced, bubbles are trapped in the polymer matrix and a cellular product is formed. The matching of the rates of gelling and foaming reactions is essential for formation of desired foams. If evolution of gas is too rapid, the foam initially expands well but then collapses because the gelling process has not proceeded enough to retain the

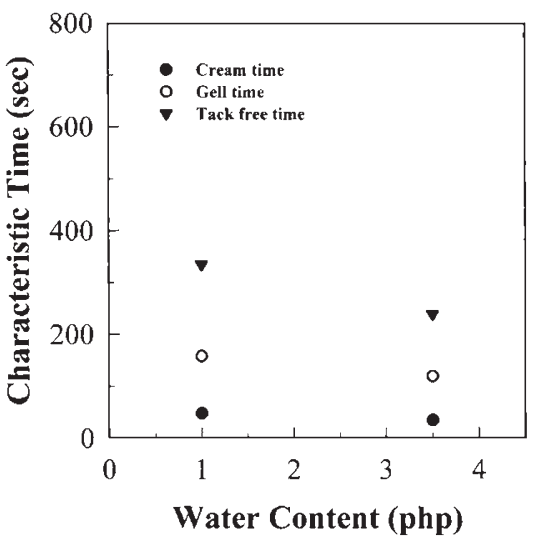

Figure 5. The dependence of the characteristic times of forming the PUF containing equal amount of the gelling catalyst on the water content.

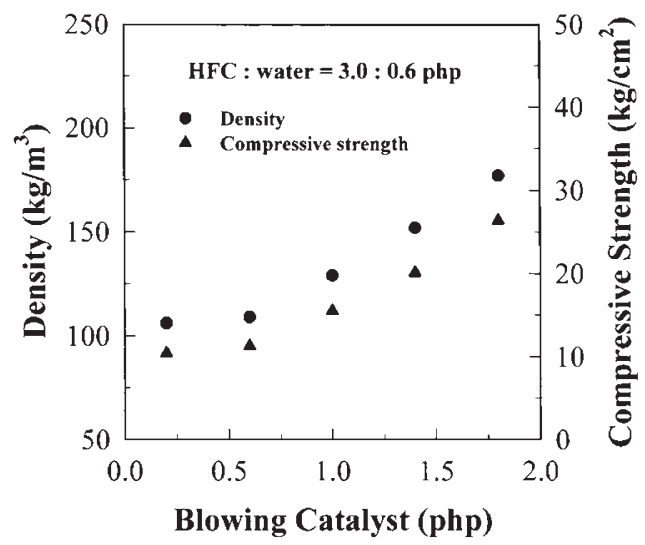

Figure 6. The density and the compressive strength of the PUF samples blown by HFC/water (4/6 mole ratio) plotted aginst the blowing catalyst content.

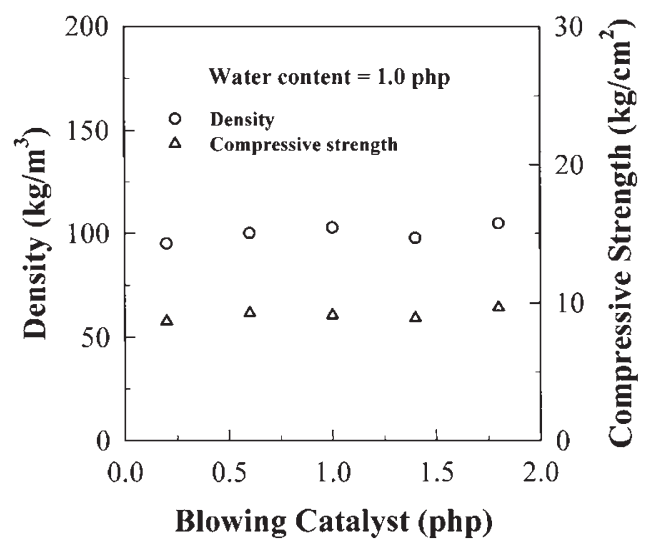

Figure 7. The density and the compressive strength of the PUF samples blown by water only plotted aginst the blowing catalyst content.

gas. $^{7-9}$ As shown in Figure 6, the added physical blowing agent such as HFC, promotes only the blowing process and hence the density and the compressive strength of the PUF samples prepared with HFC/water (4/6 mole ratio) as the blowing agents increase due 


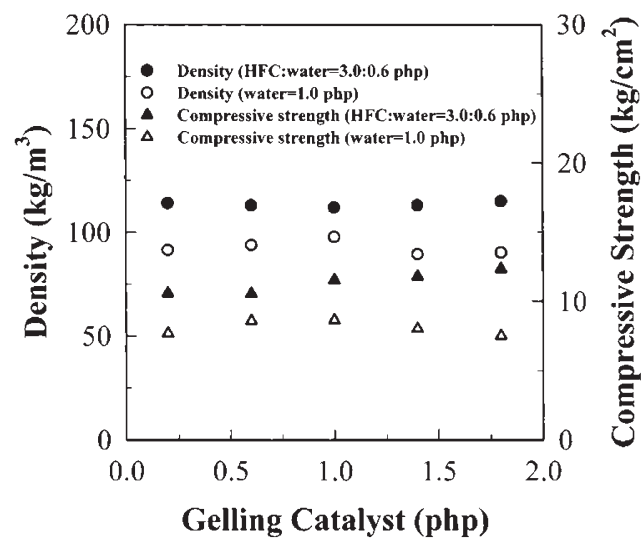

Figure 8. The density and the compressive strength the PUF samples blown by HFC/water (4/6 mole ratio) and water only plotted aginst the gelling catalyst content.

to degassing of the blowing gas by collapse of bubbles.

In the blowing process of the water only blown PUF, water reacts with the isocyanate group to generate carbon dioxide and polyurea with evolution of reaction heat. ${ }^{2,3,5-9}$ In addition, the urea group can also react with the isocyanate group to generate biuret, which introduces additional networks to the PUF. ${ }^{2,3,5-9}$ The reactions of polyurea and biuret formation are the gelling process. ${ }^{9}$ This implies that the blowing process by water accelerates the gelling process. Therefore, as shown in Figure 7, the content of the blowing catalyst does not affect significantly the density and the compressive strength of the water only blown PUF samples.

Figure 8 shows the effects of the gelling catalyst on the density and the compressive strength of the PUF samples blown by HFC/water (4/6 mole ratio) and by water only as the blowing agents. It is seen that the gelling catalyst content does not significantly influence on the density and the compressive strength of both the HFC/water (4/6 mole ratio) and the water only blown PUF samples.

Figure 9 shows the SEM micrographs of the PUF samples prepared by using (a) water only, (b) mixture of cyclopentane and water (7/3), and (c) cyclopentane only as the blowing agents. Generally the cell size is determined not only by the volatility of the blowing agents but also by various conditions such as the concentration of blowing agents, the solubility of the blowing agent, and reaction conditions. In this study, however, the PUF samples having the same density and mole of blowing gas were synthesized with water and cyclopentane as the blowing agents. The PUF samples prepared in this study were synthesized under the same operating conditions. And the chemical compositions for synthesizing the PUF samples were not changed except for the kind of blowing agent.

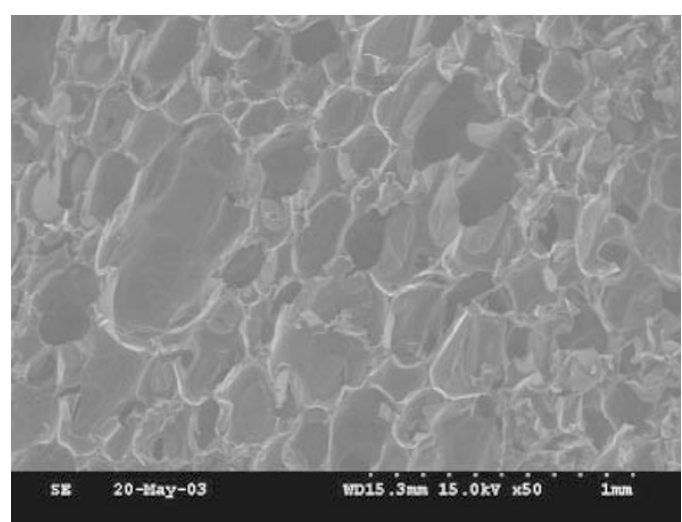

(a)

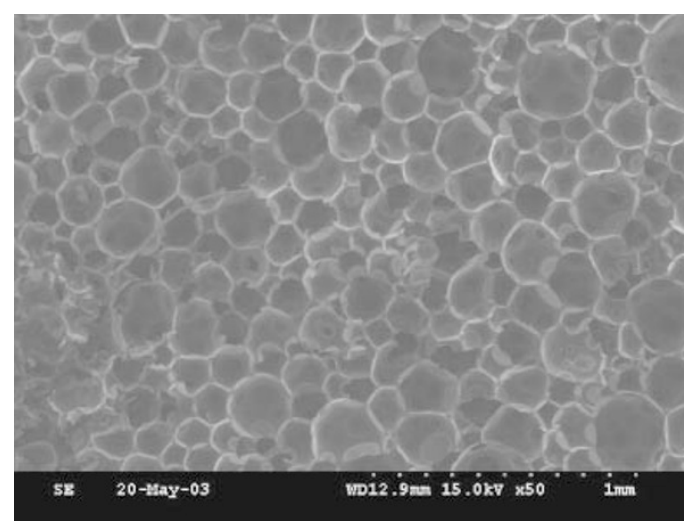

(b)

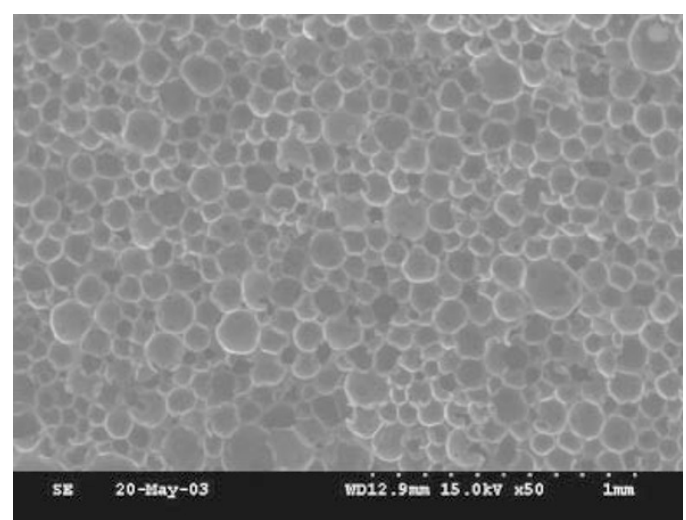

(c)

Figure 9. Scanning electron micrographs of the PUF samples blown by various blowing agents: (a) water only, (b) mixed blowing agents (cyclopentane/water $=7 / 3$, mole ratio), (c) cyclopentane only.

The solubility of $\mathrm{CO}_{2}$ in both polyol and isocyanate before polymerization can create the microcellular structure due to the solubility difference between the solutions and polyurethane polymer. However, it can be considered that the solubility of cyclopentane and $\mathrm{CO}_{2}$ in polyurethane polymer is very low at the condition (high temperature) of forming polyurethane. Therefore, the solubility difference between the cyclopentane and $\mathrm{CO}_{2}$ for the polyurethane is negligible. 
Table II. Thermal conductivity of PUF having equal density with blowing agents

\begin{tabular}{lc}
\hline Blowing agents & $\begin{array}{r}\text { Thermal conductivity } \\
\left(\mathrm{kcal} / \mathrm{mh}^{\circ} \mathrm{C}\right)\end{array}$ \\
\hline Cyclopentane & 0.0189 \\
Distilled water & 0.0197 \\
$\begin{array}{l}\text { Cyclopentane/water } \\
\quad=7 / 3 \text { (mole ratio). }\end{array}$ & 0.0184 \\
\hline
\end{tabular}

From Figure 9, it is seen that the PUF sample blown by cyclopentane (Figure 9c) or HFC has smaller cell size than the PUF sample blown by water (Figure 9a). Generally expansion of a foam is achieved by evaporation of low boiling agents or gas generation reaction of a chemical blowing agent. The polymerization and crosslinking reaction are highly exothermic. The exothermic reaction heat causes the increase of temperature of the reactants and at the vaporization point of the physical blowing agent, the physical blowing agent evaporates extremely fast. In this case, the cell size of the PUF sample becomes small., ${ }^{2,3,7}$

A chemical blowing agent generates blowing gas by the chemical reaction with the reactants. The kinetic rate of the chemical reaction increases with temperature of the reactants and the blowing gas is generated gradually. Therefore, as shown in Figure 10, the cell size of the PUF sample blown by water becomes larger than the sample blown by physical blowing agent.

The thermal conductivities of the PUF samples are listed in Table II. Generally the thermal conductivity of PUF depends on the total content of the blowing gas, thermal conductivity of entrapped blowing gas, and the structural stability of the PUF foams. ${ }^{2,3}$ The conductivities of cyclopentane and $\mathrm{CO}_{2}$ are 0.0110 and $0.0153 \mathrm{kcal} / \mathrm{mh}^{\circ} \mathrm{C}$ at $10^{\circ} \mathrm{C}$, respectively. Therefore, as shown in Table II, at the equal mole content of the blowing agents, the cyclopentane only blown PUF sample $\left(0.0189 \mathrm{kcal} / \mathrm{mh}^{\circ} \mathrm{C}\right)$ exhibits lower thermal conductivity than the water only blown PUF sample $\left(0.0197 \mathrm{kcal} / \mathrm{mh}^{\circ} \mathrm{C}\right)$. The thermal conductivity of the PUF sample blown by mixed blowing agents, however, shows lower value than the thermal conductivity of the PUF sample blown by cyclopentane only. For the mixed blowing agent, cyclopentane/water (7/3 mole ratio), the thermal conductivity of the PUF sample shows $0.0184 \mathrm{kcal} / \mathrm{mh}^{\circ} \mathrm{C}$ which is lower value than the thermal conductivity of the PUF samples blown by cyclopentane or water only. This result is probably due to the increase in the structural stability of the PUF foams.

\section{CONCLUSIONS}

The kinetic rate of forming the PUF increased with the increase of the catalyst content regardless of the types, blowing or gelling catalyst. The cell size of the PUF samples decreased with the increase of the catalyst content. The kinetic rate of forming the PUF increased with the increase in water content. This is mainly due to the reaction heat evolved by the reaction between water and PMDI. The density and the compressive strength of the PUF samples prepared with HFC/water (4/6 mole ratio) as the blowing agents increased with the content of the blowing catalyst. For the water only blown PUF samples, however, the density and the compressive strength of the PUF samples did not change significantly with the blowing catalyst. The cyclopentane only blown PUF sample had smaller cell size than the water only blown PUF sample. From this result, we concluded that the PUF sample blown by the physical blowing agents has smaller cell size than the PUF sample blown by the chemical blowing agent. The PUF sample blown by mixed blowing agent consisting of cyclopentane and water (cyclopentane/water $=7 / 3$, mole ratio) had lower thermal conductivity than the PUF sample blown by cyclopentane or water only. From this result, we suggest that the low thermal conductivity of the PUF sample blown by mixed blowing agent may be due to the increase of the structural stability of the PUF foams.

Acknowledgment. This research was supported by a grant (code BC1-102) from Carbon Dioxide Reduction \& Sequestration Research Center, one of the 21st Century Frontier Programs funded by the Ministry of Science and Technology of Korean government.

\section{REFERENCES}

1. D. Klempner and K. C. Frisch, "Handbook of Polymeric Foams and Foam Technology," Oxford University Press, New York, N.Y., 1991.

2. G. Woods, "The ICI Polyurethane Handbook," John Wiley \& Sons, New York, N.Y., 1990.

3. G. Oertel, "Polyurethane Handbook," Hanser Publisher, New York, N.Y., 1993.

4. H. C. Jung, S. C. Ryu, W. N. Kim, Y.-B. Lee, K. H. Choe, and S.-B. Kim, J. Appl. Polym. Sci., 81, 486 (2001).

5. W. J. Seo, H. C. Jung, S. H. Kim, W. N. Kim, Y.-B. Lee, K. H. Choe, and S.-B. Kim, J. Appl. Polym. Sci., 90, 12 (2003).

6. W. J. Seo, D. H. Hwang, J. H. Park, W. N. Kim, and H. S. Lee, J. Appl. Polym. Sci., 2004 (accepted).

7. K. J. Saunders, "Organic Polymer Chemistry," 2nd ed., Chapman \& Hall, New York, N.Y., 1988.

8. G. Oertel, "Polyurethane Handbook," Hanser Publisher, Munich, 1985.

9. A. Van Thuyne and B. Zeegers, J. Cell Plast., 14, 150 (1978). 